\title{
Achieving fast reconnection in resistive MHD models via turbulent means
}

\author{
G. Lapenta ${ }^{1}$ and A. Lazarian ${ }^{2}$ \\ ${ }^{1}$ Centrum voor Plasma-Astrofysica, Departement Wiskunde, Katholieke Universiteit Leuven, Celestijnenlaan 200B, \\ 3001 Leuven, Belgium \\ ${ }^{2}$ Department of Astronomy, University of Wisconsin-Madison, 475 Charter St., USA \\ Correspondence to: G. Lapenta (giovanni.lapenta@wis.kuleuven.be)
}

Received: 1 October 2011 - Revised: 29 January 2012 - Accepted: 15 February 2012 - Published: 3 April 2012

\begin{abstract}
Astrophysical fluids are generally turbulent and this preexisting turbulence must be taken into account for models of magnetic reconnection in astrophysical, solar or heliospheric environments. In addition, reconnection itself induces turbulence which provides an important feedback on the reconnection process. In this paper we discuss both the theoretical model and numerical evidence that magnetic reconnection becomes fast in the approximation of resistive MHD. We consider the relation between the Lazarian and Vishniac turbulent reconnection theory and Lapenta's numerical experiments testifying of the spontaneous onset of turbulent reconnection in systems which are initially laminar.
\end{abstract}

\section{Introduction}

Astrophysical plasmas are known to be magnetized and turbulent. Turbulence is really ubiquitous in most astrophysical environments. Turbulence in solar wind, turbulence in interstellar medium, molecular clouds are examples of wellstudied processes.

Magnetization of these fluids most frequently arises from the dynamo action to which turbulence is an essential component (see Schekochihin et al., 2007). The drivers of turbulence (e.g. supernovae explosions in the interstellar medium) inject energy at large scales and then the energy cascades down to small scales through a hierarchy of eddies spanning up over the entire inertial range. The famous Kolmogorov picture (Kolmogorov, 1941) corresponds to hydrodynamic turbulence, but, as we discuss further, a qualitatively similar turbulence also develops in magnetized fluids/plasmas. The definitive confirmation of the presence of turbulence comes from observations, e.g. observations of electron density fluc- tuations in the interstellar medium, which produce the socalled Big Power Law in the Sky (Armstrong et al., 1994; Chepurnov and Lazarian, 2010), with the spectral index coinciding with the Kolmogorov one. More direct evidence comes from the observations of spectral lines. Apart from showing non-thermal Doppler broadening, spectra of supersonic turbulent velocity fluctuations have also been revealed when analyzed with techniques like Velocity Channel Analysis (VCA) or Velocity Coordinate Spectrum (VCS) developed (see Lazarian and Pogosyan, 2000, 2004, 2006, 2008) and applied to the observational data (see Padoan et al., 2004, 2009; Chepurnov et al., 2010) rather recently.

Reconnection is a process of changing magnetic flux topology and this process has been a challenge to understand in highly conductive astrophysical fluids. As noted above, it is important to understand the process of magnetic reconnection in a realistically turbulent state of the fluid. Therefore it is important to understand the mutual feedback of turbulence and reconnection and provide the connection between the existing theory, observations and numerical experiments. This is the goal of the present paper.

We consider both the model of turbulent reconnection ${ }^{1}$ suggested in Lazarian and Vishniac (1999, henceforth LV99) and numerical evidence of fast reconnection in a few numerical papers by Lapenta and coauthors (Lapenta, 2008; Skender and Lapenta, 2010). We try to show that the two directions of the research are complementary. If LV99 mostly dealt with the situation that the turbulence already exists in the system, the work by Lapenta et al. (2008) studies the evolution of the reconnection starting with the laminar state.

\footnotetext{
${ }^{1}$ The relation between this model and earlier proposals dealing with the effects of turbulence on magnetic reconnection is discussed in more detail in Sect. 2.
} 
Taken together they can cover all the variety of practical situations. Advancing the insight into the physical relation between the two processes is the goal of the present study.

In what follows, we discuss magnetic reconnection in turbulent fluid in Sect. 2, provide the numerical confirmations of the predictions of the LV99 model in Sect. 3, consider the spontaneous onset of reconnection in MHD simulations in Sect. 4, and the role of flow pattern in Sect. 5. We discuss the implications of the expected flares of reconnection in Sect. 6 and provide our summary in Sect. 7.

\section{Reconnection can be fast in turbulent plasmas}

Astrophysical plasmas are often highly ionized and highly magnetized (Parker, 1970). Therefore, it seems natural to assume that the magnetic field is frozen-in, meaning that one disregards for all practical purposes the diffusion term in the induction equation

$$
\frac{\partial \boldsymbol{B}}{\partial t}=\nabla \times(\boldsymbol{v} \times \boldsymbol{B}-\eta \nabla \times \boldsymbol{B}),
$$

where $\boldsymbol{B}$ is the magnetic field, $\boldsymbol{v}$ is the velocity field, and $\eta$ is the resistivity coefficient. This is the concept of "frozen in" magnetic flux suggested by Alfvén (1942). It is clear, however, that magnetic flux cannot be always frozen in. Indeed, violent activity observed in the solar corona and chromosphere (Innes et al., 1997; Yokoyama and Shibata, 1995; Masuda et al., 1994; Ciaravella and Raymond, 2008) requires the presence of magnetic reconnection. Magnetic reconnection is also necessary to explain dynamo action in stars and galactic disks (Parker, 1970, 1993).

Let us start with considering the classical Sweet-Parker model of reconnection (Sweet, 1958a; Parker, 1957). In this simplest set up, two magnetic fluxes get in contact. Figure 1 shows the cross-section of the fluxes where magnetic fields change direction. The shared or guide field is, in general, present as well. In highly conducting fluid, the Ohmic diffusivity is important only over a scale $\Delta \ll L$, where $L$ is characteristic scale of the reconnecting fluxes. Both plasmas and sheared component of magnetic field must be ejected from the reconnection layer. As the ejection is limited by the Alfvén speed, one gets

$V_{\text {rec }}<V_{\mathrm{A}} \frac{\Delta}{L} \ll V_{\mathrm{A}}$.

Thus, to have fast reconnection one has to make the outflow $\Delta$ independent of resistivity. This is achieved in the LV99 model by demonstrating that in turbulent fluids, the Sweet-Parker model is getting modified and $\Delta$ gets determined by magnetic field wandering (see Fig. 1, middle plot). For the astrophysical magnetized plasmas, this is not a farfetched solution, as turbulence is ubiquitous in astrophysical environments. The turbulence arises from various instabilities acting on moving plasmas. In most cases, the turbu-
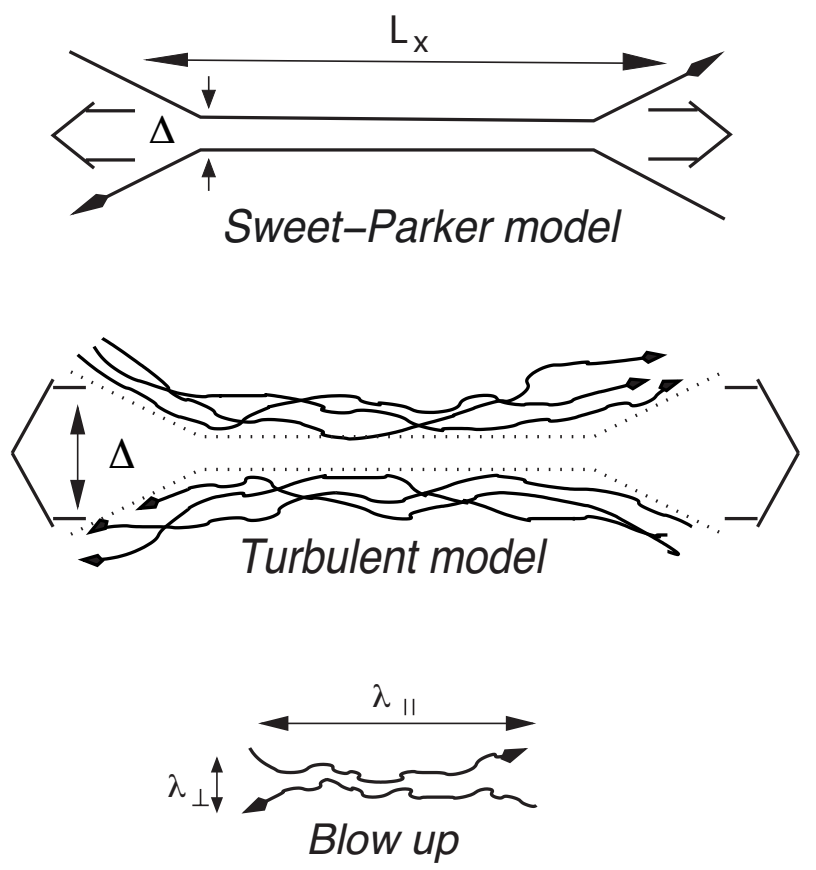

Fig. 1. Upper plot: Sweet-Parker model of reconnection. The outflow is limited by a thin slot $\Delta$, which is determined by Ohmic diffusivity. The other scale is an astrophysical scale $L \gg \Delta$. Middle plot: reconnection of weakly stochastic magnetic field according to LV99. The model accounts for the stochasticity of magnetic field lines. The outflow is limited by the diffusion of magnetic field lines, which depends on field line stochasticity. Low plot: an individual small scale reconnection region. The reconnection over small patches of magnetic field determines the local reconnection rate. The global reconnection rate is substantially larger as many independent patches come together. From Lazarian, Vishniac and Cho (2004).

lence is not caused by reconnection process, but preexisting in the astrophysical environment. For instance, in the interstellar medium supernovae explosions drive turbulence. However, for some settings, e.g. for solar flares, it is important to study how turbulence develops due to reconnection. Indeed, if magnetic reconnection were always fast, it would be not possible to accumulate and store the energy for the flare (see LV99, Lazarian and Vishniac, 2009).

The LV99 model of "stochastic reconnection", leads to reconnection speeds close to the turbulent velocity in the fluid. This speed is limited by the field wandering that determine $\Delta$. The latter changes with the scale we study reconnection at. It can be expressed through the parameters of the turbulence. Assuming isotropically driven turbulence characterized by an injection scale, $l$, smaller than the current sheet length, LV99 find

$V_{\mathrm{rec}} \approx \frac{u_{1}^{2}}{V_{\mathrm{A}}}(l / L)^{1 / 2} \approx u_{\mathrm{turb}}(l / L)^{1 / 2}$ 
where $u_{1}$ is the velocity at the driving scale and $u_{\text {turb }}$ is the velocity of the largest eddies of the strong turbulent cascade. "Strong" means only that the eddies decay through nonlinear interactions in an eddy turn over time as opposed to the regime of weak turbulence when the time of cascading is long (see LV99). In "strong MHD turbulence", motions may be very weak in the sense that the magnetic field lines are only weakly perturbed. An interesting feature of Alfvén ic turbulence is that the non-linear interactions get stronger as the turbulence cascades to small scales.

It is useful to rewrite this in terms of the power injection rate $P$. As the perturbations on the injection scale of turbulence are assumed to have velocities $u_{1}<V_{\mathrm{A}}$, the turbulence is weak at large scales. Therefore, the relation between the power and the injection velocities are different from the usual Kolmogorov estimate, namely, in the case of the weak turbulence $P \sim u_{1}^{4} /\left(l V_{\mathrm{A}}\right)$ (LV99). Thus we get,

$V_{\mathrm{rec}} \approx\left(\frac{P}{\mathrm{LV}_{\mathrm{A}}}\right)^{1 / 2} l$

where $l$ is the length of the turbulent eddies parallel to the large scale magnetic field lines as well as the injection scale.

The reconnection velocity given by Eq. (4) is obtained in MHD limit and therefore it does not depend on resistivity or plasma effects. Therefore, the LV99 model predicts that for a sufficiently high level of turbulence collisionless and collisional fluids should reconnect at the same rate. A detailed discussion of the plasma effects within the LV99 model is presented in Eyink, Lazarian and Vishniac (2011).

In some sense, the idea that turbulence can change the reconnection rate is very natural. Therefore various ideas how turbulence can increase the reconnection rate were discussed as far back as $40 \mathrm{yr}$ ago. However, these ideas fell short of solving the problem. For instance, some papers have concentrated on the effects that turbulence induces on the microphysical level. In particular, Speiser (1970) showed that in collisionless plasmas the electron collision time should be replaced with the electron retention time in the current sheet. Also Jacobson (1984) noticed that the current diffusivity should be modified to include the diffusion of electrons across the mean field due to small scale stochasticity. The exploration of these effects was important, but, they can only marginally change the reconnection rates.

The closest predecessor to LV99 was the important work of Matthaeus and Lamkin $(1985,1986)$. Those authors studied 2-D magnetic reconnection in the presence of external turbulence, both theoretically and numerically. They pointed out various turbulence mechanisms that would enhance reconnection rates, including multiple X-points as reconnection sites and motional electromagnetic fields of magnetic bubbles advecting out of the reconnection zone. The work helped to attract the attention of the community to the connection of turbulence and reconnection. However, the LV99 model introduced the importance of wandering magnetic field lines and their relevance to the analytical prediction for the reconnection speed. An enhancement of the reconnection rate was reported in both models but LV99 focused on the long term average reconnection rate. Matthaeus and Lamkin (1985, 1986), instead, included the effects of heating and compressibility as processes which can accelerate the reconnection. These effects are not included in the LV99 model or in the numerical simulations in Kowal et al. (2009) discussed below.

Some other numerical simulation papers reported evidence of fast reconnection in resistive MHD simulations. An important study of tearing instability of current sheets in the presence of background 2-D turbulence and the formation of large-scale, long-lived magnetic islands was performed in Politano et al. (1989). They present evidence for "fast energy dissipation" in 2-D MHD turbulence and show that their result does not change as they change the resolution. A more recent work of of Mininni andPouquet (2009) provides evidence for "fast dissipation" also in 3-D MHD turbulence. This phenomenon is consistent with the idea of fast reconnection, but cannot be treated as a direct evidence of the process.

\section{Fast reconnection in turbulent MHD simulations}

Testing the LV99 model presented a big computational challenge, especially at the time when the model was introduced. Studying the reconnection in 3-D was obligatory, which combined with the necessity of having magnetic fluxes turbulent, required high resolution plus long averaging in order to test the rates predicted in LV99. How to measure the reconnection rate in 3-D was not trivial either. All of this was addressed in Kowal et al. (2009) and we discuss the results of the computations there below.

The goal was testing Eq. (4). To do this, the reconnection was studied in two regions with strongly differing magnetic fields lying next to one another. The simulations were periodic in the direction of the shared field (the z-axis) and open in the reversed direction (the $\mathrm{x}$-axis). The external gas pressure was uniform and the magnetic fields at the top and bottom of the box were taken to be the specified external fields plus small perturbations to allow for outgoing waves.

To measure the speed of reconnection a new technique has been devised. Indeed, it is natural to define the reconnection rate as the rate at which the $x$ component of the magnetic field disappears. More precisely, one can consider a $y z$ slice of the simulation passing through the center of the box. Then, the rate of change of the area integral of of the absolute value $\left|B_{x}\right|$ is its flux across the boundaries of the slice minus the rate at which flux is annihilated through reconnection (see more discussion in Kowal et al., 2009)

$\partial_{t}\left(\int\left|B_{x}\right| \mathrm{d} z \mathrm{~d} y\right)=\oint \operatorname{sign}\left(B_{x}\right) \boldsymbol{E} \cdot \boldsymbol{d} \boldsymbol{l}-2 V_{\mathrm{rec}} B_{x, \operatorname{ext}} L_{z}$ 
where electric field is $\boldsymbol{E}=\boldsymbol{v} \times \boldsymbol{B}-\eta \boldsymbol{j}, B_{x \text {,ext }}$ is the absolute value of $B_{x}$ far from the current sheet and $L_{z}$ is the width of the box in the z-direction. The latter directly follows from the induction equation if one assumes that the turbulence is small amplitude and does not lead to local field reversals. Another requirement is that the stresses at the boundaries should be weak so as not to produce significant field bending at the boundaries. In other words, fields in the x-direction are coming through the top and bottom of the box, and disappear only through reconnection. The flow is not forced, instead it naturally evolves with upper and lower boundaries being open boundaries. Thus the setup reproduces the evolution of two turbulent fluxes brought together and is evolving freely as the reconnection proceeds.

Since periodic boundary conditions were assumed in the zdirection, the boundary integral on the right hand side is only taken over the top and bottom of the box. By design this definition includes contributions to the reconnection speed from contracting loops, where Ohmic reconnection has occurred elsewhere in the box and $\left|B_{x}\right|$ decreases as the end of a reconnected loop is pulled through the plane of integration. Numerical experiments showed that this estimate was roughly consistent with simply measuring the average influx of magnetic field lines through the top and bottom of the computational box and equating the mean inflow velocity with the reconnection speed. Following Eq. (5), it is possible to evaluate the reconnection speed for varying strengths and scales of turbulence and varying resistivity.

The adopted grid size in the simulations were chosen to be $256 \times 512 \times 256$ to $512 \times 1028 \times 512$ to make sure that the top and bottom of the box are sufficiently far away from the region of driven turbulence around it. More details on the adopted numerical methodology can be found in Kowal et al. (2009).

Transient effects are expected as fluxes are brought together. To compensate for them it, is desirable to allow the system to relax to the Sweet-Parker reconnection initially. This was accomplished by allowing the system to evolve for seven Alfvén crossing times without turbulent forcing. As subsequently the isotropic turbulent forcing was turned on in a volume centered in the midplane (in the xz plane) of the simulation box and extending outwards by a quarter of the box size. The turbulence reached its full amplitude in around eight crossing times and was stationary thereafter.

In Fig. 2 the current density in an xy slice of the computational box with fully developed turbulence is shown. One can notice that the narrow stationary current sheet characteristic of Sweet-Parker reconnection is replaced by a chaotic structure with numerous narrow peaks in the current density. The outflow region $\Delta$ (see Eq. 2) gets wider than in the Sweet-Parker case. Numerous faint features indicate reconnection between adjacent turbulent eddies and oppositely moving magnetic loops described in LV99.

In Fig. 3 the results of numerical experiments with varying amounts of input turbulence driving power are shown. The
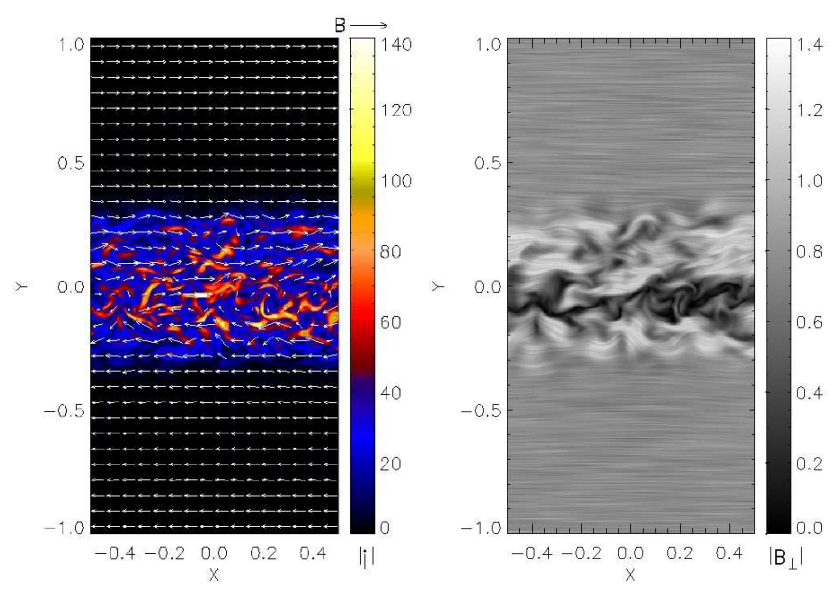

Fig. 2. Left panel: current intensity and magnetic field configuration during stochastic reconnection. We show a slice through the middle of the computational box in the xy plane after twelve dynamical times for a typical run. The shared component of the field is perpendicular to the page. The intensity and direction of the magnetic field is represented by the length and direction of the arrows. The color bar gives the intensity of the current. The reversal in $B_{x}$ is confined to the vicinity of $y=0$ but the current sheet is strongly disordered with features that extend far from the zone of reversal. Right panel: representation of the magnetic field in the reconnection zone with textures.

line drawn through the simulation points corresponds to the predicted scaling of reconnection rate with the square root of the input power (see Eq. 4).

In Fig. 4 the results for different fluid resistivities are shown. The input turbulence driving power and scale are fixed. In this case the resistivity $\eta$ is taken to be uniform, except near the edges of the computational grid where it falls to zero over five grid points. This was done to mitigate edge effects arising for large values of the resistivity. As benchmark Fig. 4 also presents results for laminar reconnection that scale as $\sqrt{\eta}$, which is the scaling for Sweet-Parker reconnection. The reconnection in the presence of turbulence is fast, as predicted in LV99. Indeed, the reconnection rate is insensitive to the values of fluid resistivity.

To get insight into the importance of plasma effects, reconnection was also studied with the anomalous resistivity, i.e. with the resistivity that increases with the current densities. This enhances the local speed of individual reconnection events but results in Kowal et al. (2009) testify that the total reconnection rate does not change by the presence of anomalous resistivity. This also corresponds to the LV99 predictions.

In addition, the study of numerical effects is presented in Lazarian et al. (2011). Numerical resistivity decreases with the increase of the numerical resolution. Therefore, if the numerical effects were influencing the reconnection rate, the increase of the resolution would decrease the reconnection rates. This is not what is seen in simulations (Lazarian et al., 


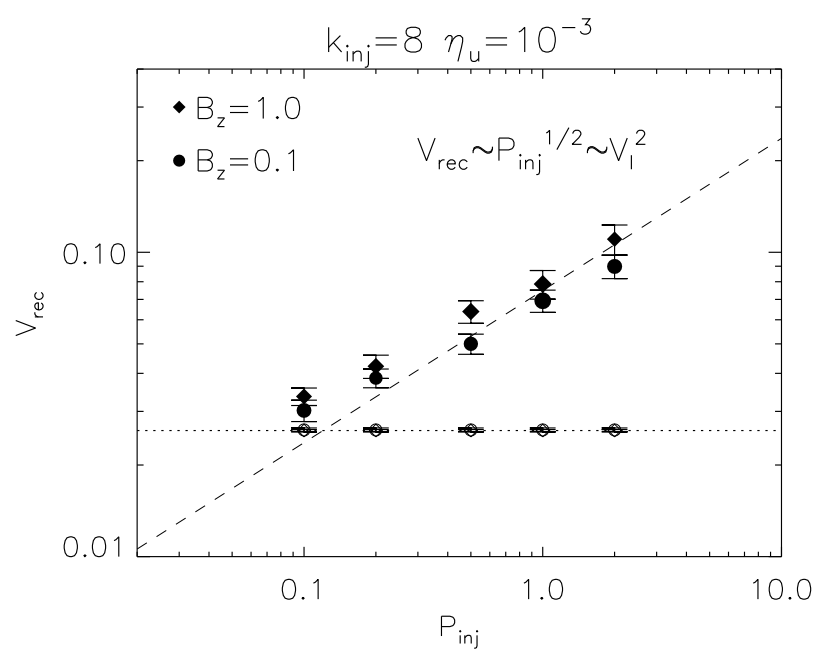

Fig. 3. Reconnection speed versus input power for the driven turbulence. We show the reconnection speed, defined by Eq. (4) plotted against the input power for an injection wavenumber equal to 8 (i.e. a wavelength equal to one eighth of the box size) and a resistivity $v_{u}$. The dashed line is a fit to the predicted dependence of $P^{1 / 2}$ (see Eq. 3). The horizontal line shows the laminar reconnection rates for each of the simulations before the turbulent forcing started. Here uncertainty in the time averages is indicated by the size of the symbols and the variances are shown by the error bars. The initial strength of $B_{x}$ is 1 .

2011). If anything, the reconnection rates slightly increase with the increase of resolution, which is due to the fact that the turbulence proceeds to smaller scales. As a result, field wandering over smaller scales is available and therefore the outflow region gets slightly thicker as the numerical viscosity decreases.

Finally, it is important to give a few words in relation to our turbulence driving. We drive our turbulence solenoidally, i.e. in the incompressible fashion (see Cho and Lazarian, 2003), to minimize the effects of compression, which does not play a role in the LV99 model. The turbulence driven in the volume around the reconnection layer corresponds to the case of astrophysical turbulence, which is also volumedriven. On the contrary, the case of the turbulence driven at the box boundaries would produce spatially inhomogeneous imbalanced turbulence for which we do not have analytical predictions (see discussion of such turbulence in Beresnyak and Lazarian, 2009). We stress that it is not the shear size of our numerical simulations but the correspondence of the observed scalings to those predicted in LV99 that allows us to claim that we proved that the 3-D reconnection is fast in the presence of turbulence.

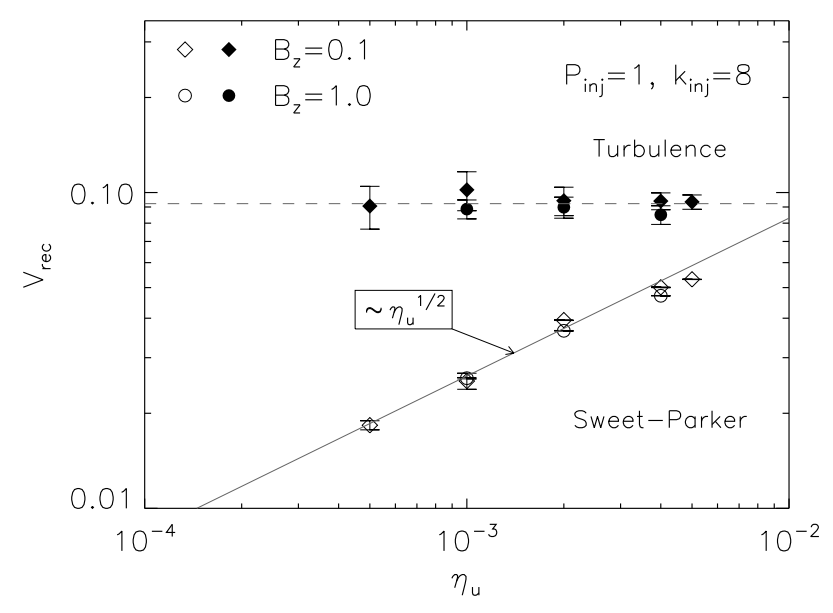

Fig. 4. Reconnection speed versus resistivity. We show the reconnection speed plotted against the uniform resistivity of the simulation for an injection wavenumber of 8 and an injected power of one. We include both the laminar reconnection speeds, using the hollow symbols, fit to the expected dependence of $\eta_{u}$, and the stochastic reconnection speeds using the filled symbols. As before, the symbol sizes indicate the uncertainty in the average reconnection speeds and the error bars indicate the variance. We included simulations with large, $B_{z}=1$, and small, $B_{z}=0.1$, guide fields.

\section{Spontaneous onset of turbulent reconnection in laminar system}

Macroscopically laminar systems can spontaneously transition toward unsteady regimes characterised by faster rates and presenting features that are closely related to the turbulent reconnection regimes described above.

The long-recognized natural pathway for reconnection in laminar systems is the Sweet-Parker (SP) regime (Sweet, 1958a; Parker, 1963). In SP reconnection, a thin and elongated current layer forms separating two areas of opposing magnetic polarity. The plasma and the field flows towards the current layer in the direction normal to the elongated side and outflows along the axis of the current. Figure 5a shows the typical flow pattern.

The SP current layer, like any other current layer, is in itself unstable to the tearing mode (Biskamp, 1993, 2000) producing secondary islands. The presence of the flow pattern and the finite length of the layer prevent a direct application of the textbook analysis of the tearing mode. The first studies of the instability of SP layers were reported by Bulanov et al. (1979) and are summarised in recent textbooks (Biskamp, 1993, 2000). The conclusion was that for aspect ratios of the SP layer (length of the current channel, $\Delta$ over its width $\delta$ ) exceeding approximately 100 , the SP layer becomes unstable. The aspect ratio of a SP layer is directly determined by the resistivity in the system. Using the parametrization of the resistivity with the Lundquist number $S$, the aspect ratio is simply $\Delta / \delta=\sqrt{S}$ (Priest and Forbes, 2000). It follows 


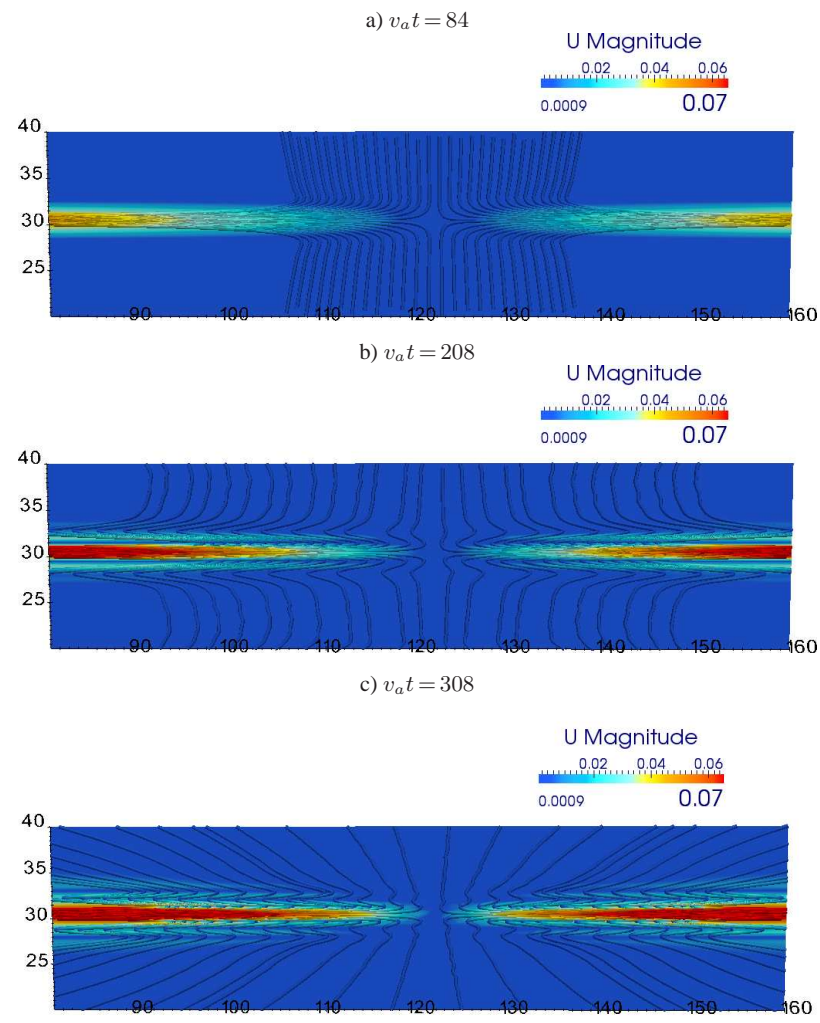

Fig. 5. Early evolution (xz plane). The flow lines are shown superimposed over a false colour representation of the flow speed. The SP layer first forms and the flow pattern around it changes its nature. Three times are shown. Blow up of the central region, the full box is $240 \times 60$.

that given a sufficiently low resistivity, corresponding to a Lundquist number in excess of $S=10^{4}$, a SP will become naturally unstable to formation of secondary islands.

Low resistivity was not easily accessible to simulations until recently. At low resolutions, the numerical resistivity exceeds the intended low resistivity preventing the exploration of truly low resistive regimes. However, in recent years truly low resistivity regimes became accessible thanks to modern numerical methods and state of the art computational facilities. Great progress has been made in understanding this spontaneous transition from laminar SP reconnection to an unsteady regime characterised by a random onset of secondary islands.

The configuration of a SP layer is intrinsically 2-D and requires a 2-D analysis (Ni et al., 2010). Furthermore the SP layer is embedded in macroscopic configurations that further affect the stability and evolution (Schindler and Birn, 1999). However, a reduced 1-D analysis (Loureiro et al., 2007) can still take into account the effects of the flow present in the SP layer. The flow has a stabilising effect (Bulanov et al., 1978) that prevents the onset for aspect ratios below approximately 100 , but at higher aspect ratios (corresponding to higher Lundquist numbers) the instability is present. The growth rate of the secondary instability is found to scale as $\gamma \approx S^{1 / 4}$ and the fastest growing mode corresponds to a number of islands that scales as $S^{3 / 8}$ (Huang and Bhattacharjee, 2010). The power is positive: the growth rate of the secondary instability is faster for lower resistivity. Additionally as the resistivity is lowered and the aspect ratio is increased, the number of magnetic islands increases. The emerging picture from linear theory is then clear: as the Lundquist number is increased the SP layer becomes progressively more elongated, with a higher aspect ratio. The more elongated the SP layer becomes, the larger the number of secondary islands becomes, and the faster they grow after forming. A clear indication of transition towards a turbulent regime like that is covered in the previous sections.

The prediction of the linear theory with respect to the number and growth rate of secondary islands have been tested and verified in especially designed simulations (Samtaney et al., 2009; Cassak et al., 2009; Bhattacharjee et al., 2009) .

Similarly, the threshold for onset ( $S$ approximately $10^{4}$ ) has been also confirmed (Skender and Lapenta, 2010). Additionally, the simulation studies have proven that turbulence, if present, has a direct impact on the process. The presence of pre-existing fluid turbulence changes the onset of the secondary island instability (Loureiro et al., 2009; Skender and Lapenta, 2010). A SP layer still forms also in the presence of moderate levels of turbulence but it is more prone to become unstable. The stronger seed reduces the threshold for the onset and the system transitions more quickly to an unsteady regime of reconnection characterized by multiple islands and reconnection sites. Indeed, in all simulations the instability of the SP layer is seeded by numerical noise. Spectral methods avoid such noise completely and the instability cannot develop (Ng and Ragunathan, 2011) unless a seed in the form of a low level of turbulence is added explicitly.

An important consequence of the formation of secondary islands and the onset of unsteady reconnection is the acceleration of the reconnection process (Lapenta, 2008). Reconnection is usually defined as fast when two conditions are met.

First, the reconnection rate should be independent of the mechanism that allows the decoupling of the plasma (and specifically the electrons in the case of advanced two fluid and kinetic models). In resistive MHD, the mechanisms allowing such decoupling are resistivity and, possibly, viscosity. With the onset of secondary islands, simulations observe not only an increased rate of reconnection but also a reconnection rate that is independent of resistivity. As noted in Sect. 3, careful studies varying the resistivity in the system have shown that the rate becomes insensitive or even independent of resistivity altogether (Lapenta, 2008; Loureiro et al., 2009; Huang and Bhattacharjee, 2010; Uzdensky et al., 2010).

Second, for reconnection to be fast in absolute terms, its rate must be a significant fraction of the local Alfvén speed measured with the density and magnetic field strength at the 
entry of the reconnection region $v_{\mathrm{A} \text {,in }}$. For a reconnecting Harris sheet, Skender and Lapenta (2010) report a rate of approximately $\Delta \dot{\Psi} / v_{\mathrm{A}, \text { in }} B_{\text {in }} \approx .015$ with peaks of twice that value. Note that the value reported by Skender and Lapenta (2010) is computed with a different normalisation Alfvén speed based on the peak rather than the inflow density that is a factor of 10 smaller. Here we prefer to translate the result in terms of to the actual Alfvèn speed of the inflowing plasma for better comparison with the other litarature. For force free equlibria, the peak reconnection rate also nearly reaches $\Delta \dot{\Psi} / v_{\mathrm{A}, \text { in }} B_{\text {in }} \approx .015$ (Skender and Lapenta, 2010). Similarly, in the case of the reconnection induced by the coalescence of two magnetic islands, Huang and Bhattacharjee (2010) report a reconnection rate of $\Delta \dot{\Psi} / v_{\mathrm{A}} B_{0} \approx .01$, in agreement with the case of the Harris sheet. The reconnection rate of unsteady MHD resistivity is therefore somewhat lower than that typical of the fastest kinetic regimes (peak rate of $\Delta \dot{\Psi} / v_{\mathrm{A}, \text { in }} B_{\text {in }} \approx .1$, Birn and et al., 2001) but is still very strong.

Many of the properties of the fast reconnection process following the onset of the secondary islands instability can be understood with a simplified approach based on the properties of the tearing mode (Bhattacharjee et al., 2009). For an initial current sheet with a magnetic field profile

$\mathbf{B}(z)=B_{0} \tanh (z / a) \hat{\mathbf{x}}$

the linear theory based on reduced MHD with uniform resistivity $\eta$ predicts a maximum growth rate (Schindler, 2006, p. 242):

$\gamma_{\max } a / v_{\mathrm{A}}=0.623 \cdot\left(\mu_{0} a v_{\mathrm{A}} / \eta\right)^{-1 / 2}$

corresonding to the wave number:

$k_{\max } a=1.358 \cdot\left(\mu_{0} a v_{\mathrm{A}} / \eta\right)^{-1 / 4}$

In the case of the stability of a SP layer, one can simply use the same result but consider that the initial state is itself the SP layer, with a thickness that is proportional to the square of the resistivity, $a=\eta^{1 / 2}$. It immediately follows that the fastest growing mode of the secondary island instability scales as $k \propto \eta^{-3 / 8}$ and the corresponding growth rate as $\gamma \propto \eta^{-1 / 4}$. These simple estimates agree remarkably with the more in depth analysis by Loureiro et al. (2007); Huang and Bhattacharjee (2010), reported above.

The argument can be carried further in the non-linear regime to estimate the non-linear reconnection rate during the subsequent growth of the islands. In the case of the tearing mode, the island growth is governed by the Rutherford equation (Rutherford, 1973), stating that the size of the magnetic islands $\left(w_{\mathrm{I}}\right)$ grows in time linearly (rather than exponentially). The temporal derivative of the size of a magnetic island is $\dot{w}_{\mathrm{I}} \propto \eta \Delta^{\prime} / \mu_{0}$ (Kadomtsev, 1992, p. 76), where, for the initial configuration assumed above,

$\Delta^{\prime}=\frac{2}{a}\left(\frac{1}{k a}-k a\right)$
At long wavelengths, where $k a \ll 1, \Delta^{\prime} \propto a^{-2}$, and assuming again the initial thickness to be that of the SP layer, the Rutherford equation leads to a rate of island growth independent of resistivity, $\dot{w}_{\text {I }} \propto \eta^{0}$ (i.e. it is independent of $\eta$ ).

Note that the dependence on $k$ is not considered in the scaling argument because the secondary islands are observed in the simulation to coalesce and the value of $k$ is expected to settle on a value constrained more by the macroscopic system rather than by the fastest growing linear mode.

Rutherford's theory is valid in the assumption that the the resistive time is slower that the hydrodynamic time: $\tau_{\mathrm{R}} / \tau_{\mathrm{H}}>1$ (Rutherford, 1973). In the present case, this assumption remains valid at all scales because at each secondary reconnection site the spatial scales become smaller and both hydrodynamic and resitive times are reduced. Since both times are reduced in proportion of the reduced thickness of the reconnecting layer, their ratio $\tau_{\mathrm{R}} / \tau_{\mathrm{H}}>1$ remains large.

\section{Role of the flow pattern in unsteady reconnection}

A consequence of the onset of the secondary island instability is the profound modification of the plasma flow patterns in the reconnection region. To investigate the flow, a specific simulation is used. The initial state is a Harris sheet with magnetic field given by Eq. (6), uniform initial temperature corresponding to $v_{\mathrm{s}} / v_{\mathrm{A}}=1$ and density

$\rho=\rho_{0} / \cosh ^{2}(z / a)$

A density bachground is added with $\rho_{\mathrm{b}}=\rho_{0} / 10$ (resulting in a initially slighty non-uniform temperature). The evolution is followed with visco-resistive MHD, using the FLIP3D-MHD code (Brackbill, 1991). Note that here and in the rest of the 3-D simulations with FLIP3D-MHD, the coordinate system has $z$ in the direction of the initial gradients (not $y$ ).

The simulations have uniform resistivity corresponding to a Lundquist number, $S=\eta_{0} a v_{\mathrm{A}} / \eta=10^{4}$ and a viscosity corresponding to a Reynolds number, $R=\rho_{0} v_{\mathrm{A}} a / \eta=$ $10^{4}$. All results are presented in normalised units where the magnetic field is normalised to $B_{0}$, the density to $\rho_{0}$, time with the Alfvén time $\tau_{\mathrm{A}}=a / v_{\mathrm{A}}$, space with the initial current sheet thickness $a$ and velocity with the Alfvén speed $v_{\mathrm{A}}=B_{0} /\left(\mu_{0} \rho_{0}\right)^{1 / 2}$. The simulation box has sides $L_{x} / a=240$ and $L_{z}=60$. The code is $3-\mathrm{D}$ but the $y$ dependence is suppressed.

During the SP regime, the flow is primarily directed normal to the sides of the SP layer. In the two inflow sides, the flow is vertical, and in the two outflow sides it becomes horizontal, veering within the SP layer. Figure 5a shows such a configuration for the present simulation. The actual flow in the simulation is just as the typical textbook SP cartoon (Biskamp, 2000).

As the simulation evolves, the flow pattern changes and becomes primarily concentrated near the separatrices. This is a typical feature of the flow in presence of magnetic islands 
due to the tearing instability. Figure $5 \mathrm{~b}$ and $\mathrm{c}$ demonstrate the transition. An x-point is forming in the center as a consequence of the secondary island instability. The flow pattern becomes now just like the textbook cartoon of the flow near an X-point in the tearing mode (Biskamp, 2000).

Still later times are shown in Fig. 6. After the onset of the secondary island instability, progressively more secondary islands and x-points are formed. The new formed islands coalesce with pre-existing islands and migrate with the flow towards the two ends of the simulation box (Lapenta, 2008). The present simulation uses periodic boundary conditions but the same series of events develops also with open boundary conditions (Skender and Lapenta, 2010; Bettarini and Lapenta, 2010) with the only difference that the flow carries the formed islands outside the open boundaries.

The presence of multiple islands causes the flow to form characteristic loops. In a previous paper (Lapenta, 2008), the name conveyor belts was used to explain the role of such flow loops. The flow assumes a pattern where the flow lines that come out of the reconnection process bend and return towards the same reconnection region. The effect is similar to that of recursive reconnection (Parnell et al., 2008) observed in magnetic skeleton configurations representative of solar coronal configurations. In recursive reconnection the outflow of one reconnection site feeds into another. In the present simulation the same recursive process is also observed. In Fig. $6 \mathrm{c}$ the outflow from the $\mathrm{x}$-point located at $x / a=115$ feeds the reconnection process at the $\mathrm{x}$-point located at $x / a=90$ (and the similarly by symmetry on the other side). But more conspicuously, the same reconnection $\mathrm{x}$-point at $x / a=115$ feeds itself with most flow lines going out of it and coming back forming a conveyor belt.

In accordance with the theoretical predictions above, for reconnection to be fast the flow must become stronger in the unsteady regime. Comparing the scales in Fig. 5 with Fig. 6 , the flow accelerates strongly. Figure 7 shows separately the inflow component along $z$ and the outflow component along $x$. The outflow is at the Alfvén speed and the inflow is a significant fraction of it. At steady state the inflow speed equals the reconnection electric field and is by definition the reconnection rate.

The concept of streamlines and in particular of conveyor belts should not be confused with actual recirculation of plasma. The flow pattern is distinct from the matter actually flowing. On those stepping into rivers staying the same other and other waters flow (Heraclitus of Ephesus, 2001, DK22B12, Fragment 91). The flow can present the same pattern over a certain period but the plasma elements going through the flow are other and other. The streamlines are instant photos of the velocity field at a given instant. They are obtained here with the paraview software using the fourthfifth order Runge-Kutta ODE solver that traces the lines everywhere tangent to the velocity field at that instant. But an actual fluid element feels at every instant the local speed at that instant. A moment later it feels another speed. Flow

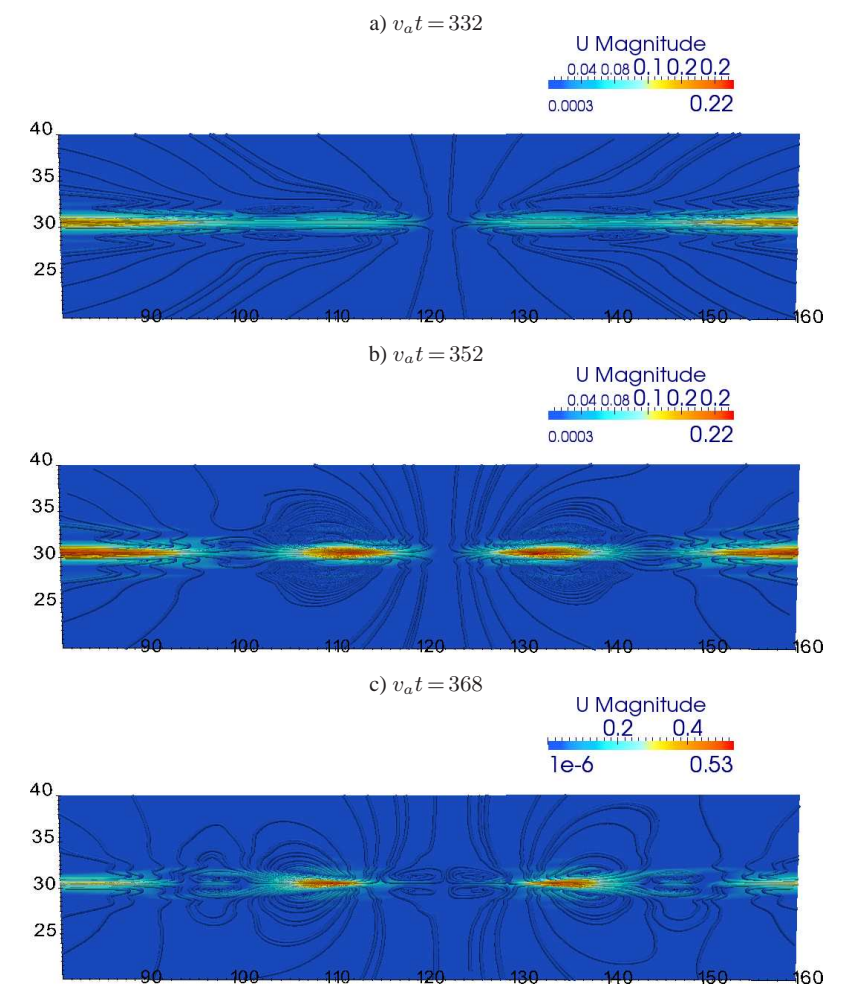

Fig. 6. Later evolution (xz plane). The flow lines are shown superimposed over a false colour representation of the flow speed. The SP layer is destabilised by the secondary island instability. Three times are shown. The first two have the same color scale, but the third has higher values to avoid saturation. Blow up of the central region, the full box is $240 \times 60$.

lines are not the same thing as particle trajectories. To analyse actual trajectories of fluid elements we use Lagrangian markers. Lagrangian markers are real particles of the fluid that move with the local instantaneous velocity just as a physical fluid would. These are not kinetic particles moving with their own speed but rather fluid elements moving with the local average flow speed. The FLIP3D-MHD code is based on a dual grid-particle representation that includes the presence of Lagrangian markers (Brackbill, 1991).

Figure 8 shows a selected number of trajectories of Lagrangian markers. The initial starting point at time $t=0$ in the simulation is chosen appropriately to identify particles that will go through multiple reconnection sites. Some trajectories only pass reconnection once: those are particles that are drawn toward the neutral line $(z / a=30)$ and then ejected away from it (reddish trajectories on the left and light cyan on the right). However, there are other classes of trajectories (green on the left and magenta on the right) that are first drawn towards the neutral line and after being pushed away, come back a second time towards the neutral line and then are finally ejected away from it. These are particles that are captured in the conveyor belts and are pushed towards 


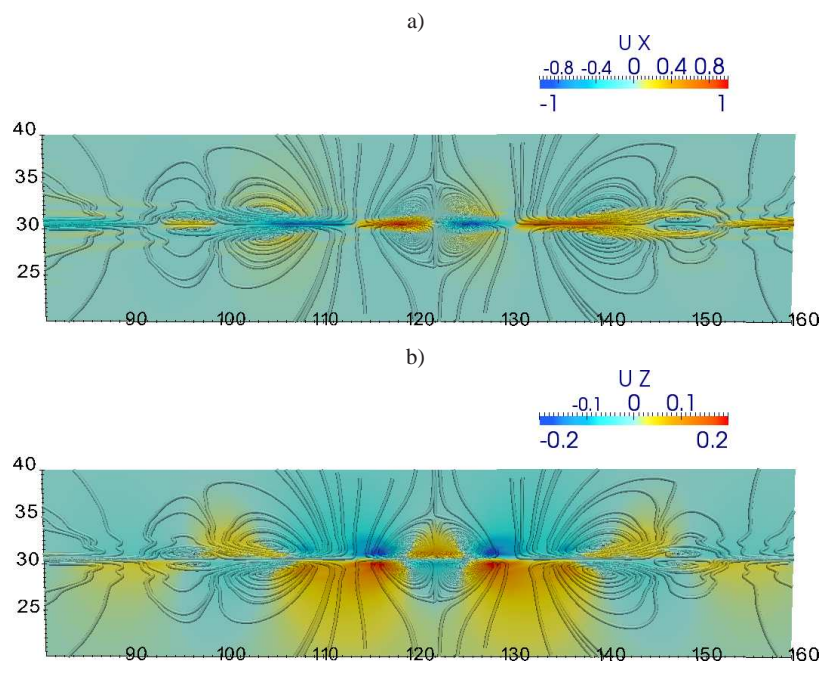

Fig. 7. Flow pattern at time $t / \tau_{\mathrm{A}}=375.5$. The outflow component $U_{x}$ is shown in panel a and the inflow component $z$ in (b). Blow up of the central region, the full box is $240 \times 60$.

a reconnection site and then away from it and then towards another one again. Note, however, that these trajectories are still monotonic in the horizontal direction: the same particle does not turn back to go towards the same x-point. For example on the left of center, the markers first take part to reconnection at the site located near $x / a=115$ and then at $x / a=90$. No particle is detected to go through the same reconnection site twice. Just as the citation by Heraclitus suggested. This conclusion is consistent with the observation of closed stream function loops because the streamfunction is a photograph of the flow at a given time, while the trajectory of Lagrangian markers take time into account. As the fluid elements move, the islands move, grow and merge. The time for particles to move along their path is significant compared with the island evolution, resulting in trajctories that are different from the streamfunction patterns.

\section{Discussion}

A magnetic field embedded in a laminar perfectly conducting fluid preserves its topology for all time (Parker, 1979). Although ionized astrophysical objects, like stars and galactic disks, are almost perfectly conducting, they show indications of changes in topology, "magnetic reconnection", on dynamical time scales (Parker, 1970; Lovelace, 1976; Priest and Forbes, 2002). Reconnection can be observed directly in the solar corona (Innes et al., 1997; Yokoyama and Shibata, 1995; Masuda et al., 1994), but can also be inferred from the existence of large scale dynamo activity inside stellar interiors (Parker, 1993; Ossendrijver, 2003). Solar flares (Sturrock, 1966) and $\gamma$-ray busts (Fox et al., 2005; Galama et al., 1998) are usually associated with magnetic reconnection.

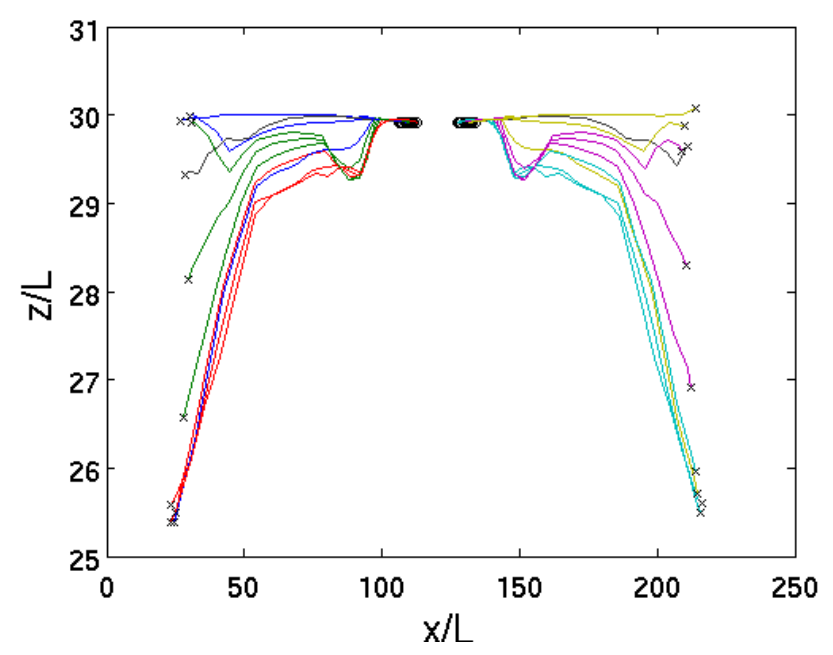

Fig. 8. Trajectory of Lagrangian markers. Miltiple trajectories are shown in different colours. Each trajectory starts at a circle and ends at a cross. The initial positions for the aprticles are equallys paced between $x-L_{x}= \pm 7 L$ and $x-L_{x} / 2= \pm 14 L$ on either side from the center of the $\mathrm{x}$-axis and at vertical position $z / L=29.9167$, just a little below the central neutral line.

Previous work has concentrated on showing how reconnection can be rapid in plasmas with very small collisional rates (Shay et al., 1998; Drake, 2001; Drake et al., 2006; Daughton et al., 2006), which substantially constrains astrophysical applications of the corresponding reconnection models.

In comparison, our results point out to an attractive universal astrophysical solution for the magnetic reconnection: magnetic reconnection in presence of turbulence is always fast independent of the collisional or collisionless state of plasmas. The formal criterion of the applicability of the LV99 model based on the MHD approximation to realistic plasmas is discussed in Eyink, Lazarian and Vishniac (2011).

Our paper provides a connection between the LV99 theory and numerical simulations demonstrating the spontaneous onset of fast reconnection. Indeed, according the LV99 model the reconnection in the initially laminar state is slow. The outflow, however, incites turbulence in the system. This turbulence induces faster reconnection and a wider outflow gets more turbulent. This should induce a positive feedback process which results in the flare of reconnection. This mechanism is central to the theoretical explanation of the spontaneous onset of fast reconnection observed in papers by Lapenta et al. (2008).

The spontaneous onset of reconnection is likely to be the cause of the solar flares. The flares require the accumulation of magnetic flux of opposite polarity prior to the flare. The accumulation means low reconnection rate, which is readily achived for the observed mean level of turbulence in solar atmosphere (Eyink et al., 2011). As the flux is accumulated, the effects that we described in this paper are expected to 
come into place and a abrupt release of energy through magnetic reconnection is expected. A similar process can take place during gamma ray busts (Lazarian et al., 2002; Zhang and Yan, 2011). A relatively low level of turbulence is a prerequisite for the flares. In the presence of high amplitude turbulence, reconnection is going to be fast from the very beginning, preventing the accumulation of magnetic flux at the pre-flare stage.

Observationally, predictions of the LV99 model for flares are in rough agreement with the estimates of the thickness of the outflow regions obtained in observations (Ciravella and Raymond, 2008). In addition, the initiation of the reconnection by Alfvenic perturbations predicted in LV99 was confirmed by observations in Sych et al. (2009). Direct observations of turbulent reconnection in other environments, e.g. in interstellar medium are not feasible at the moment. However, the indirect evidence of LV99-type reconnection comes from the observations of magnetic fields in molecular clouds and accretion disks. In fact, on the basis of LV99 model Lazarian (2005) predicted the process of magnetic field removal termed "reconnection diffusion". Numerical studies of reconnection diffusion (see Santo de Lima et al., 2010, 2011) deliver results on expected magnetic flux diffusion that are consistent with observations.

The caveat here is that the LV99 model is the 3-D model and it appeals to the 3-D wandering of magnetic field lines. We believe that the fact that acceleration was observed also in 2-D systems, as shown in Sect. 5, testifies that the modification of LV99 model is applicable to the systems of reduced dimentionality. It is discussed in Eyink et al. (2011) that the existence of the "rough" turbulent velocity field should induce the Richarson diffusion and therefore the effective magnetic field wandering. The corresponding theory have not been developed for the 2-D turbulence. However, results on the acceleration of reconnection in 2-D simulations in the presence of turbulence (Lapenta, 2008; Loureiro et al., 2009; Huang and Bhattacharjee, 2010; Uzdensky et al., 2010) suggest that the results of LV99 may apply to 2-D systems.

Our paper provides an alternative to the interpretation of astrophysical reconnection within laminar reconnection models. We showed that pre-existing turbulence as well as turbulence induced by the process of magnetic reconnection itself can dramatically change the reconnection rates. Interestingly enough, the small - scale reconnection events may be determined by small scale physics, but the total reconnection rates may still be determined by the turbulent level in the system.

\section{Summary}

The results of the present paper can be briefly summarised as follows:

1. The LV99 theory of fast turbulent reconnection and more recent results on the spontaneous onset of turbu- lent reconnection (Lapenta, 2008; Loureiro et al., 2009; Huang and Bhattacharjee, 2010; Uzdensky et al., 2010) are related to each other.

2. Fast turbulent reconnection is critically linked with selffeeding flow patterns and with the feedback by turbulence induced by the outflow on the reconnection process.

3. The numerical results reviewed above suggest that flares of magnetic reconnection, e.g. explaining solar flares, may be explained on the basis of pure resistive MHD.

4. Our work testifies in favor of a widely applicable model of astrophysical reconnection that is independent of subtle plasma effects. At the same time, it provides an alternative view to attempts to model astrophysical reconnection without including the effects of turbulence.

Acknowledgements. The research of AL is supported by the NSF grant AST 0808118 and the Center for Magnetic Self Organization in Laboratory and Astrophysical Plasmas (CMSO). The present work is supported in part by the NASA MMS mission, by the Onderzoekfonds KU Leuven (Research Fund KU Leuven) and by the European Commission's Seventh Framework Programme (FP7/2007-2013) under the grant agreement no. 218816 (SOTERIA project, www.soteria-space.eu) and no. 263340 (SWIFF project, www.swiff.eu). The simulations were conducted on the resources of the NASA Advanced Supercomputing Division (NAS), of the The NASA Center for Computational Sciences Division (NCCS), and of the Vlaams Supercomputer Centrum (VSC) at the Katholieke Universiteit Leuven.

Edited by: J. Sears

Reviewed by: two anonymous referees

\section{References}

Alfvén, H.: Existence of Electromagnetic-Hydrodynamic Waves, Nature, 150, 405-406, 1942.

Armstrong, J. W., Rickett, B. J. and Spangler, S. R.: Electron density power spectrum in the local interstellar medium, Astrophys. J., 443, 209-221, 1995.

Beck, R., Brandenburg, A., Moss, D., Shukurov, A., and Sokoloff, D.: Galactic Magnetism: Recent Developments and Perspectives, Annu. Rev. Astronomy and Astrophysics, 34, 155-206, 1996.

Beresnyak, A. and Lazarian, A.: Structure of Stationary Strong Imbalanced Turbulence, Astrophys. J., 702, 460-471, 2009.

Bettarini, L. and Lapenta, G.: Spontaneous non-steady magnetic reconnection within the solar environment, Astron. Astrophys., 518, A57+, doi:10.1051/0004-6361/200913652, 2010.

Bhattacharjee, A., Huang, Y.-M., Yang, H., and Rogers, B.: Fast reconnection in high-Lundquist-number plasmas due to the plasmoid Instability, Physics of Plasmas, 16, 112102 , doi:10.1063/1.3264103, 2009.

Birn, J., Drake, J. F., Shay, M. A., Rogers, B. N., Denton, R. E., Hesse, M., Kuznetsova, M., MA, Z. W., Bhattacharjee, A., Otto, A., and Pritchett, P. L.: Geospace Environmental Modeling 
(GEM) magnetic reconnection challenge, J. Geophys. Res., 106, 3715-3719, 2001.

Biskamp, D.: Magnetic reconnection via current sheets, Physics of Fluids, 29, 1520-1531, 1986.

Biskamp, D.: Nonlinear Magnetohydrodynamics, Cambridge University Press, Cambridge, 1993.

Biskamp, D.: Magnetic Reconnection in Plasmas, Cambridge University Press, Cambridge, 2000.

Brackbill, J.: FLIP MHD: A particle-in-cell method for magnetohydrodynamics, J. Comp. Phys., 96, 163-192, 1991.

Brandenburg, A. and Subramanian, K.: Astrophysical magnetic fields and nonlinear dynamo theory, Physics Reports, 417, 1209, 2005

Bulanov, S. V., Syrovatskii, S. I., and Sakai, J.: Stabilizing influence of plasma flow on dissipative tearing instability, Soviet Journal of Experimental and Theoretical Physics Letters, 28, 177-183, 1978.

Bulanov, S. V., Sakai, J., and Syrovatsii, S. I.: Tearing-mode instability in approximately steady MHD configurations, Sov. J. Plasma Phys., 5, 157-167, 1979.

Cassak, P. A., Shay, M. A., and Drake, J. F.: Scaling of SweetParker reconnection with secondary islands, Physics of Plasmas, 16, 120702, doi:10.1063/1.3274462, 2009.

Chepurnov, A., Lazarian, A.: Extending the Big Power Law in the Sky with Turbulence Spectra from Wisconsin $\mathrm{H} \alpha$ Mapper Data, Astrophys. J., 710, 853-858, 2010.

Chepurnov, A., Lazarian, A., Stanimirović, S., Heiles, C., and Peek, J. E. G.: Velocity Spectrum for H I at High Latitudes, Astrophysical Journal, 714, 1398-1406, 2010.

Cho, J. and Lazarian, A.: Compressible magnetohydrodynamic turbulence: mode coupling, scaling relations, anisotropy, viscosity?damped regime and astrophysical implications, Mont. Not. Royal. Astron. Soc., 345, 325-339, 2003.

Ciaravella, A. and Raymond, J. C.: The Current Sheet Associated with the 2003 November 4 Coronal Mass Ejection: Density, Temperature, Thickness, and Line Width, Astrophys. J., 686, 1372-1382, 2008.

Cummings, A. C. and Stone, E. C.: Anomalous Cosmic Rays and Solar Modulation, Space Sci. Rev., 83, 51-62, 1998.

Daughton, W., Scudder, J., and Karimabadi, H.: Fully kinetic simulations of undriven magnetic reconnection with open boundary conditions, Physics of Plasmas, 13, 072101, doi:10.1063/1.2218817, 2006.

Daughton, W., Roytershteyn, V., Albright, B. J., Bowers, K., Yin, L., and Karimabadi, H.: Reconnection Dynamics in SemiCollisional Plasmas, AGU Fall Meeting Abstracts A1705, 2008.

de Gouveia dal Pino, E. M. and Lazarian, A.: Production of the large scale superluminal ejections of the microquasar GRS $1915+105$ by violent magnetic reconnection, Astronomy and Astrophysics, 441, 845-853, 2005.

Draine, B. T. and Lazarian, A.: Electric Dipole Radiation from Spinning Dust Grains, Astrophys. J., 508, 157-179, 1998.

Drake, J. F.: Magnetic explosions in space, Nature, 410, 525-526, 2001.

Drake, J. F., Swisdak, M., Che, H., and Shay, M. A.: Electron acceleration from contracting magnetic islands during reconnection, Nature, 443, 553-556, 2006.

Drake, J. F., Opher, M., Swisdak, M., and Chamoun, J. N.: A Magnetic Reconnection Mechanism for the Generation of Anomalous
Cosmic Rays, Astrophys. J., 709, 963-974, 2010.

Elmegreen, B. G. and Scalo, J.: Interstellar Turbulence I: Observations and Processes, Annu. Rev. Astronomy and Astrophysics, 42, 211-273, 2004.

Eyink, G. L., Lazarian, A., and Vishniac, E. T.: Fast Magnetic Reconnection and Spontaneous Stochasticity, Astrophys. J., 743, 51, 2011.

Fisk, L. A. and Gloeckler, G.: The Common Spectrum for Accelerated Ions in the Quiet-Time Solar Wind, Astrophys. J., 640, L79-L82, 2006.

Florinski, V. and Zank, G. P.: Particle acceleration at a dynamic termination shock, Geophys. Res. Lett., 33, 15110, doi:10.1029/2006GL026371, 2006.

Fox, D. B., Frail, D. A., Price, P. A., et al.: The afterglow of GRB 050709 and the nature of the short-hard $\gamma$-ray bursts, Nature, 437, 845-850, 2005.

Galama, T. J., Vreeswijk, P. M., van Paradijs, J., et al.: An unusual supernova in the error box of the $\gamma$-ray burst of 25 April 1998 , Nature, 395, 670-672, 1998.

Haverkorn, M., Brown, J. C., Gaensler, B. M., and McClureGriffiths, N. M.: The Outer Scale of Turbulence in the Magnetoionized Galactic Interstellar Medium, Astrophys. J., 680, 362370, 2008.

Heraclitus of Ephesus: Fragments: the collected wisdom of Heraclitus, Viking, New York, 2001.

Huang, Y.-M. and Bhattacharjee, A.: Scaling laws of resistive magnetohydrodynamic reconnection in the high-Lundquist-number, plasmoid-unstable regime, Physics of Plasmas, 17, 062104, doi:10.1063/1.3420208, 2010.

Kadomtsev, B.: Tokamak Plasma: A Complex Physical System, IOP Publishing, Bristol, 1992.

Kolmogorov, A.: The Local Structure of Turbulence in Incompressible Viscous Fluid for Very Large Reynolds' Numbers, Akademiia Nauk SSSR Doklady, 30, 301-305, 1941,

Kowal, G., Lazarian, A., Vishniac, E. T., and Otmianowska-Mazur, K.: Numerical tests of fast reconnection in weakly stochastic magnetic fields, Astrphys. J. , 700, 63, doi:10.1088/0004637X/700/1/63, 2009.

Kowal, G., de Gouveia Dal Pino, E. M., and Lazarian, A.: Magnetohydrodynamic Simulations of Reconnection and Particle Acceleration: Three-dimensional Effects, Astrphys. J., 735, 102, doi:10.1088/0004-637X/735/2/102, 2011.

Kowal, G., de Gouveia Dal Pino, E., and Lazarian, A.: On the role of magnetic reconnection in jet/accretion disk systems, Phys. Rev. Lett., submitted, 2012.

Innes, D. E., Inhester, B., Axford, W. I., and Wilhelm, K.: Bidirectional plasma jets produced by magnetic reconnection on the Sun, Nature, 386, 811-813, 1997.

Jacobson, A. R. and Moses, R. W.: Nonlocal dc electrical conductivity of a Lorentz plasma in a stochastic magnetic field, Phys. Rev. A, 29, 3335-3342, 1984.

Jokipii, R.: Cosmic Rays in Interstellar Turbulence, Interstellar Turbulence, 70-78, available at: http://ebooks. cambridge.org/chapter.jsf?bid=CBO9780511564666\&cid= CBO9780511564666A017, 1999.

Jokipii, J. R. and Giacalone, J.: The Theory of Anomalous Cosmic Rays, Space Sci. Rev., 83, 123-136, 1998.

Lapenta, G.: Self-Feeding Turbulent Magnetic Reconnection on Macroscopic Scales, Phys. Rev. Lett., 100, 235001, 
doi:10.1103/PhysRevLett.100.235001, 2008.

Lazarian, A.: Astrophysical Implications of Turbulent Reconnection: from cosmic rays to star formation, Magnetic Fields in the Universe: From Laboratory and Stars to Primordial Structures, AIP Conference Series, 784, 42-53, 2005.

Lazarian, A. and Opher, M.: A Model of Acceleration of Anomalous Cosmic Rays by Reconnection in the Heliosheath, Astrophys. J., 703, 8-21, 2009.

Lazarian, A. and Pogosyan, D.: Velocity Modification of H I Power Spectrum, Astrophys. J., 537, 720-748, 2000.

Lazarian, A. and Pogosyan, D.: Velocity Modification of the Power Spectrum from an Absorbing Medium, Astrophys. J., 616, $943-$ 965, 2004.

Lazarian, A. and Pogosyan, D.: Studying Turbulence Using Doppler-broadened Lines: Velocity Coordinate Spectrum, Astrophys. J., 652, 1348-1365, 2006.

Lazarian, A. and Pogosyan, D.: Studying Velocity Turbulence from Doppler-broadened Absorption Lines: Statistics of Optical Depth Fluctuations, Astrophys. J., 686, 350-362, 2008.

Lazarian, A. and Vishniac, E. T.: Reconnection in a Weakly Stochastic Field, Astrophys. J., 517, 700-718, 1999.

Lazarian, A., Vishniac, E. T., and Cho, J.: Magnetic Field Structure and Stochastic Reconnection in a Partially Ionized Gas, Astrophys. J., 603, 180-197, 2004.

Loureiro, N. F., Schekochihin, A. A., and Cowley, S. C.: Instability of current sheets and formation of plasmoid chains, Physics of Plasmas, 14, 100703, doi:10.1063/1.2783986, 2007.

Loureiro, N. F., Uzdensky, D. A., Schekochihin, A. A., Cowley, S. C., and Yousef, T. A.: Turbulent magnetic reconnection in two dimensions, Mon. Not. Royal Astron. Soc., 399, L146-L150, doi:10.1111/j.1745-3933.2009.00742.x, 2009.

Lovelace, R. V. E.: Dynamo model of double radio sources, Nature, 262, 649-652, 1976.

Masuda, S., Kosugi, T., Hara, H., Tsuneta, S., and Ogawara, Y.: A loop-top hard X-ray source in a compact solar flare as evidence for magnetic reconnection, Nature, 371, 495-497, 1994.

Matthaeus, W. H. and Lamkin, S. L.: Rapid magnetic reconnection caused by finite amplitude fluctuations, Phys. Fluids, 28, 303307, 1985.

Matthaeus, W. H. and Lamkin, S. L.: Turbulent magnetic reconnection, Phys. Fluids, 29, 2513-2534, 1986.

McComas, D. J. and Schwadron, N. A.: An explanation of the Voyager paradox: Particle acceleration at a blunt termination shock, Geophys. Res. Lett., 33, 4102, doi:10.1029/2005GL025437, 2006.

McKee, C. F. and Ostriker, E. C.: Theory of Star Formation, Annu. Rev. Astronomy and Astrophysics, 45, 565-687, 2007.

Mininni, P. D. and Pouquet, A.: Finite dissipation and intermittency in magnetohydrodynamics, Phys. Rev. E, 80, 025401, doi:10.1103/PhysRevE.80.025401, 2009.

Moraal, H., Caballero-Lopez, R. A., McCracken, K. G., McDonald, F. B., Mewaldt, R. A., Ptuskin, V., and Wiedenbeck, M. E.: Cosmic ray energy changes at the termination shock and in the heliosheath, Physics of the Inner Heliosheath, 858, 219-225, 2006.

Ng, C. S. and Ragunathan, S.: High Lundquist Number Resistive MHD Simulations of Magnetic Reconnection: Searching for Secondary Island Formation, ArXiv e-prints, 2011.

Ni, L., Germaschewski, K., Huang, Y.-M., Sullivan, B. P., Yang, H., and Bhattacharjee, A.: Linear plasmoid instability of thin current sheets with shear flow, Physics of Plasmas, 17, 052109, doi:10.1063/1.3428553, 2010.

Ossendrijver, M.: The solar dynamo, Astronomy and Astrophysics Review, 11, 287-367, 2003.

Padoan, P., Juvela, M., Kritsuk, A., and Norman, M. L.: The Power Spectrum of Supersonic Turbulence in Perseus, Astrophys. J., 653, L125-L128, 2006.

Parker, E. N.: Sweet's Mechanism for Merging Magnetic Fields in Conducting Fluids, J. Geophys. Res., 62, 509-520, 1957.

Parker, E. N.: The Solar-Flare Phenomenon and the Theory of Reconnection and Annihiliation of Magnetic Fields, Astrophys. J. Suppl., 8, 177, doi:10.1086/190087, 1963.

Parker, E. N.: The Generation of Magnetic Fields in Astrophysical Bodies. I. The Dynamo Equations, Astrophys. J., 162, 665-673, 1970.

Parker, E. N.: Cosmical magnetic fields: Their origin and their activity, Oxford, Clarendon Press; New York, Oxford University Press, 1979, 858, 1979.

Parker, E. N.: A solar dynamo surface wave at the interface between convection and nonuniform rotation, Astrophys. J., 408, 707719, 1993.

Parnell, C., Haynes, A., and Galsgaard, K.: Recursive Reconnection and Magnetic Skeletons, Astrophys. J., 675, 1656, doi:10.1086/527532, 2008.

Petschek, H. E.: Magnetic field annihilation, in: The Physics of Solar Flares. Proceedings of the AAS-NASA Symposium, edited by: Hess, W. H., 28-30 October, 1963, at the Goddard Space Flight Center, Greenbelt, MD, USA NASA Special Publication 425, 1964.

Politano, H., Pouquet, A., and Sulem, P. L.: Inertial ranges and resistive instabilities in two-dimensional magnetohydrodynamic turbulence, Physics of Fluids B, 1, 2330-2339, 1989.

Priest, E. and Forbes, T.: Magnetic Reconnection : MHD Theory and Applications, Cambridge University Press, Cambridge, 2000.

Priest, E. R. and Forbes, T. G.: The magnetic nature of solar flares, Astronomy and Astrophysics Review, 10, 313-377, 2002.

Rutherford, P. H.: Nonlinear growth of the tearing mode, Physics of Fluids, 16, 1903-1908, doi:10.1063/1.1694232, 1973.

Samtaney, R., Loureiro, N. F., Uzdensky, D. A., Schekochihin, A. A., and Cowley, S. C.: Formation of Plasmoid Chains in Magnetic Reconnection, Phys. Rev. Lett., 103, 105004, doi:10.1103/PhysRevLett.103.105004, 2009.

Schekochihin, A. A., Cowley, S. C., and Dorland, W.: Interplanetary and interstellar plasma turbulence, Plasma Physics and Controlled Fusion, 49, 195, doi:10.1088/0741-3335/49/5A/S16, 2007.

Schindler, K.: Physics of Space Plasma Activity, Cambridge University Press, Cambridge, doi:10.2277/0521858976, 2006.

Schindler, K. and Birn, J.: Thin current sheets and magnetotail dynamics, J. Geophys. Res., 1042, 25001-25010, doi:10.1029/1999JA900258, 1999.

Shay, M. A. and Drake, J. F.: The role of electron dissipation on the rate of collisionless magnetic reconnection, Geophys. Res. Lett., 25, 3759-3762, 1998.

Shibata, K. and Tanuma, S.: Plasmoid-induced-reconnection and fractal reconnection, Earth Planets and Space, 53, 473-482, 2001.

Shimizu, T., Kondo, K., Ugai, M., and Shibata, K.: Magnetohy- 
drodynamics Study of Three-Dimensional Fast Magnetic Reconnection for Intermittent Snake-Like Downflows in Solar Flares, Astrophys. J., 707, 420-427, 2009.

Skender, M. and Lapenta, G.: On the instability of a quasiequilibrium current sheet and the onset of impulsive bursty reconnection, Physics of Plasmas, 17, 022905, doi:10.1063/1.3299326, 2010.

Speiser, T. W.: Conductivity without collisions or noise, Planetary Space Sci., 18, 613-622, 1970.

Stix, M.: The galactic dynamo, Astronomy and Astrophysics, 42, 85-89, 1975.

Sturrock, P. A.: Model of the High-Energy Phase of Solar Flares, Nature, 211, 695-697, 1966.

Sweet, P.: The production of high energy particles in solar flares, Il Nuovo Cimento (1955-1965), 8, 188-196, doi:10.1007/BF02962520, 1958a.
Sweet, P. A.: The Neutral Point Theory of Solar Flares, Electromagnetic Phenomena in Cosmical Physics, 6, 123-133, 1958b.

Uzdensky, D. A., Loureiro, N. F., and Schekochihin, A. A.: Fast Magnetic Reconnection in the PlasmoidDominated Regime, Phys. Rev. Lett., 105, 235002, doi:10.1103/PhysRevLett.105.235002, 2010.

Yokoyama, T. and Shibata, K.: Magnetic reconnection as the origin of X-ray jets and $\mathrm{H} \alpha$ surges on the Sun, Nature, 375, 42-44, 1995.

Zhang, M.: Acceleration of galactic and anomalous cosmic rays in the heliosheath, Physics of the Inner Heliosheath, 858, 226-232, 2006. 